\title{
PRÉSENCE EN FRANCE (DORDOGNE, PYRÉNÉES ORIENTALES, HAUTE-SAVOIE, BAS-RHIN, PAS DE CALAIS, MANCHE ET COTES DU NORD) DE LA FORME SPINOSUM DOBY ET DEBLOCK, 1957, DE SIMULIUM ORNATUM MEIGEN, 1818 (DIPTÈRES-SIMULIIDÉS)
}

\author{
Par J.-M. DOBY et F. DAVID
}

Cette forme, décrite par l'un de nous (Doby et Deblock, 1957), et caractérisée, à l'état nymphal, par la présence de tubercules tégumentaires épineux et en très grand nombre (alors que la forme type n'en présente que de rares et discoïdes), n'était connue jusqu'ici que d'une seule station, dans le Tyrol autrichien (Langen). A la fin de la description de cette forme, la possibilité de trouver des stations en France par examen systėmatique, effectué à un grossissement suffisant, des téguments nymphaux, était envisagée.

Depuis, l'examen de matériel français d'origines diverses est venu confirmer cette opinion. Le caractère, sinon fréquent, du moins non rare, de cette forme, est maintenant démontré. Nous l'avons en effet récoltée dans les stations suivantes :

\section{Dordogne}

1) St-Pardoux : 24-3-1959. Altitude : $200 \mathrm{~m}$. Ruisseau de 20 à $50 \mathrm{~cm}$. de large, de profondeur variable; courant rapide; en compagnie de $S$. latipes et de S. hirtipes var. arvernense.

2) La Coquille : 24-3-1959. Altitude : $350 \mathrm{~m}$. Ruisseau de un mètre de large, de 20 à $30 \mathrm{~cm}$. de profondeur; fond de cailloux ; courant rapide ; végétation peu abondante; en compagnie de $S$. monticola, $S$. reptans et $S$. hirtipes var. arvernense.

3) Nontron 1 : 23-3-1959. Altitude : $200 \mathrm{~m}$. Ruisseau de $1 \mathrm{~m}$. à $1 \mathrm{~m}$. 50 de large, de profondeur très variable (de 5 à $50 \mathrm{~cm}$.) ; fond de grosses pierres, couvertes de fontinales ; courant rapide ; en compagnie de $S$. hirtipes var. arvernense, $S$. latipes, $S$. ornatum type et S. decorum. 
4) Nontron 2: 23-3-1959. Altitude : $200 \mathrm{~m}$. Ruisseau de $30 \mathrm{~cm}$. de large et de quelques centimètres de profondeur, très encombré de végétation; courant rapide; en compagnie de $S$. ornatum type et de S. latipes.

5) Miallet : 24-3-1959. Altitude : $300 \mathrm{~m}$. Petit ruisseau dans le fond d'un fossé, de $30 \mathrm{~cm}$. de large et de quelques centimètres de profondeur ; végétation variable; fond de cailloux et graviers ; courant modéré ; en compagnie de $S$. latipes et $S$. ornatum type.

\section{Pyrénées-Orientales}

6) Olette: 6-9-1958. Altitude : $700 \mathrm{~m}$. Petite rigole d'irrigation sous taillis, de $20 \mathrm{~cm}$. de large sur quelques centimètres de profondeur; bords très encaissés, avec végétation couvrant le lit et traînant un peu dans le courant rapide et tumultueux; fond de pierres plates ; en compagnie de $S$. lamachi (1), $S$. latipes et $S$. ornatum type.

\section{Haute-Savoie}

7) Habère-Lullin : 10-8-1959. Altitude : $800 \mathrm{~m}$. Ruisseau de $30 \mathrm{~cm}$. de large sur $5 \mathrm{~cm}$. de profondeur, à bords très encaissés; fond de cailloux; très peu de végétation; courant rapide; eau très riche en sels de calcium (ruisseau pétrifiant); en compagnie de $S$. monticola.

\section{Bas-Rhin}

8) Hohwald : 24-7-1959. Altitude : $475 \mathrm{~m}$. Ruisseau l' « Andlau »; un mètre de large sur 10 à $50 \mathrm{~cm}$. de profondeur; lit encombré de grosses pierres; courant violent; végétation peu abondante; en compagnie de $S$. variegatum.

\section{Pas-de-Calais}

9) Warlincourt: 23-7-1953. Altitude : $100 \mathrm{~m}$. Ruisseau «La Quilienne »; $60 \mathrm{~cm}$. de large sur $10 \mathrm{~cm}$. de profondeur ; fond de terre et pierres; végétation en abondance moyenne; courant moyen.

\section{Manche}

10) Quettreville: 21-2-1959. Altitude : $50 \mathrm{~m}$. Ruisseau de $80 \mathrm{~cm}$. de large sur $20 \mathrm{~cm}$. de profondeur; fond sableux, végétation d'abondance moyenne ; courant modéré ; en compagnie de $S$. ornatum type.

(1) $S$. lamachi Doby et David, 1959 : espèce caractérisée notamment, à l'état nymphal, par un appareil respiratoire constitué. de chaque côté, de deux filaments seulement, très épais et boudinés (Vie et Milieu, sous presse). 


\section{Gôtes-du-Nord}

11) St-Lunaire : 17-3-1957. Altitude : $20 \mathrm{~m}$. Ruisseau « L'Arguenon 》; $1 \mathrm{~m}$. 50 de large, sur 10 à $25 \mathrm{~cm}$, de profondeur; fond de sable et cailloux ; très peu de végétation ; courant assez fort ; en compagnie de $S$. ornatum type, $S$. latipes et $S$. aureum.

Cette forme semble donc ne pas constituer une rareté. Elle est cependant beaucoup moins fréquente que la forme type. Voici, par exemple, la proportion de stations de $S$. ornatum spinosum par rapport à celles de la forme type (ou de la variété nitidifrons), observées dans une même région :

\begin{tabular}{|c|c|c|}
\hline Régions de France & $\begin{array}{c}\text { Nombre de stations } \\
\text { de la forme spinosum }\end{array}$ & $\begin{array}{c}\text { Nombre de stations } \\
\text { de l'espèce-type et de } \\
\text { la var. nitidifrons }\end{array}$ \\
\hline Massif-Central .... & 5 & 11 \\
\hline Pyrénées ......... & 1 & 6 \\
\hline Alpes...$\ldots \ldots \ldots$ & 1 & 1 \\
\hline Est $\ldots \ldots \ldots \ldots \ldots$ & 1 & 6 \\
\hline Nord $\ldots \ldots \ldots \ldots$ & 1 & 19 \\
\hline Ouest..$\ldots \ldots \ldots$. & 2 & 25 \\
\hline
\end{tabular}

Dans la limite de nos observations et jusqu'à plus ample informé, cette forme semble relativement plus fréquente dans les régions de moyenne altitude qu'en plaine.

Toutes les stations que nous avons relevées étaient constituées par des petits ruisseaux, de $1 \mathrm{~m} .50$ de large au grand maximum.

Le fait que cette forme ait été relativement fréquemment trouvée (6 fois) associée à la forme type confirme bien l'opinion antérieurement émise, selon laquelle le caractère épineux des tubercules tégumentaires et leur nombre ne semblent pas liés à des conditions biologiques particulières. 


\section{BIBLIOGRAPHIE}

Doby (J. M.) et David (F.), 1959. - Complément à l'étude des Simuliidés (Diptères, Nématocères) du Nord de la France. Bull. Soc. Path. Exot., 52, 208.

Doвy (J. M.) et Deblock (S.), 1957. - Présence de tubercules tégumentaires épineux chez la nymphe d'une forme autrichienne de Simulium ornatum Meigen, 1818 : S. ornatum f. spinosum n. f. Ann. Parasit. hum. et comp., 32,328 .

Dову (J. M.) et Doвy-Duвois (M.), 1955. - Complément à la forme des Simuliidés des Pyrénées-Orientales. Observations sur le cocon de Simulium ornatum Meigen 1818 et de sa variété nitidifrons Edwards 1920. Vie et Milieu, 6, 123.

Grenier (P.), 1953. - Simuliida de France et d'Afrique du Nord. Encyclopédie entomologique, Lechevalier édit., Paris.

\section{(Laboratoire de Parasitologie}

de la Faculté de Médecine et de Pharmacie de Rennes) 